\title{
Entrevista
}

\section{Diego Molano \\ Vega: \\ ¿Colombia \\ en retroceso?}

DOI: 10.29236/sistemas.n156a3

Maneja el mapa completo del país, lo observa, sugiere y recomienda. Describe las carencias y plantea soluciones.

\section{Sara Gallardo M}

Diego Molano Vega, exministro de Tecnologías de la Información y las Telecomunicaciones (TIC), consultor internacional de gobiernos y empresas en transformación digital ubicado en Washington, presidente de la universidad Área Andina, asesor senior del Banco Interamericano de Desarrollo (BID), de Mckinsey y de la OECD, entre tantas otras responsabilidades, tiene el país completo en su ejercicio profesional.
No lo desvelan esas múltiples ocupaciones ni el otro montón como investigador de varios centros de pensamiento en los Estados Unidos. Le quita el sueño la educación de sus hijas de dos y cuatro años en estos tiempos de pandemia.

"Ellas son muy chiquitas y a los 10 minutos están fatigadas con cualquier actividad inspirada en tecnología. Las escuelas están cerradas posiblemente hasta febrero y no 


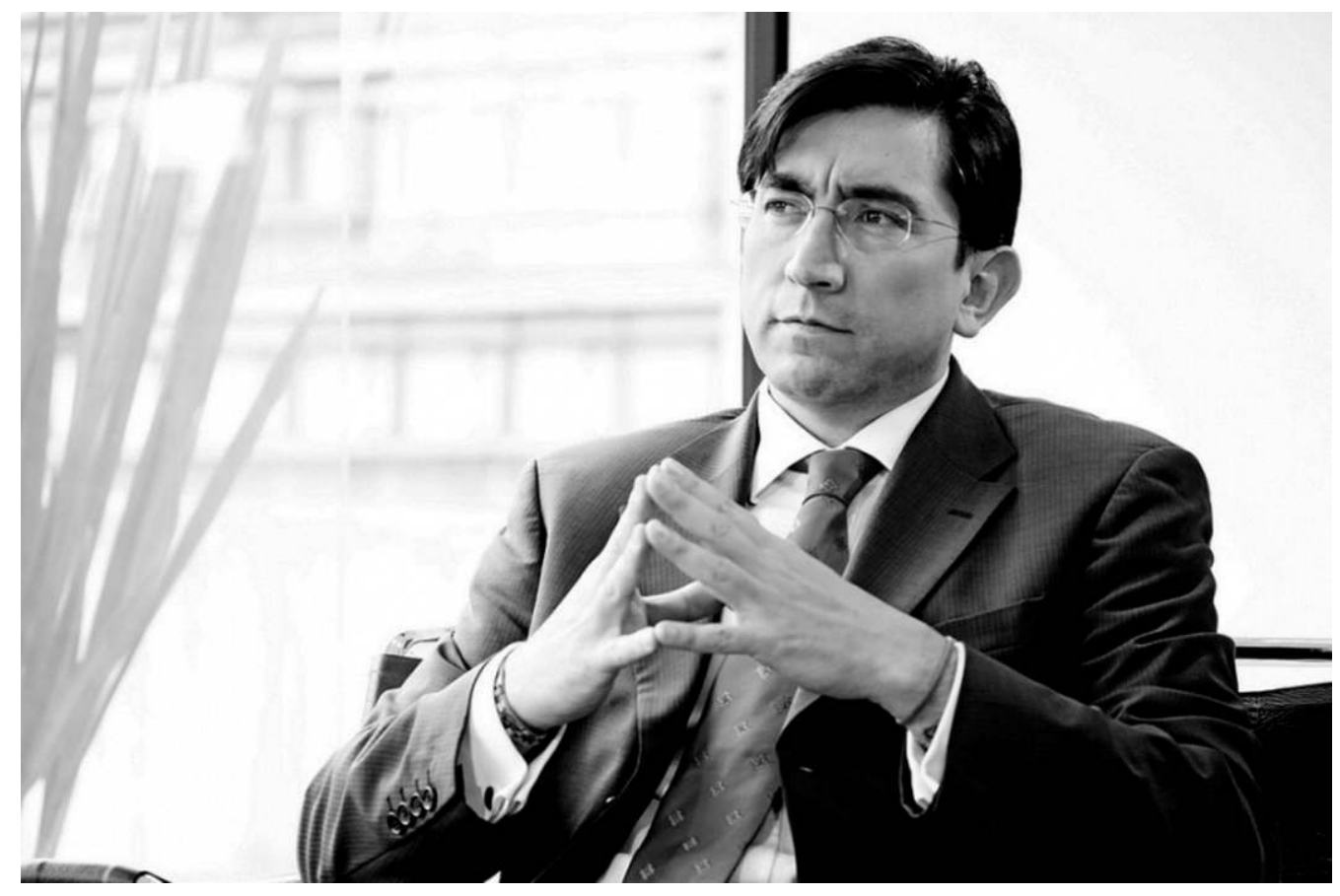

podemos parar su educación seis o siete meses, así que el reto es inmenso", por esa razón uno de sus proyectos inmediatos apunta a la creación de una escuela para formación de padres.

Reconocido en el mundo como una de las 100 personas más influyentes en asuntos de gobierno digital y el número 20 en ambientes Fintech de Iberoamérica, atendió esta entrevista con la sencillez del boyacense y la firmeza de un profesional muy experimentado y conocedor del sector como ninguno.

Razones suficientes para dejarlo hablar sobre Colombia desde todas sus aristas, enfatizar en las carencias y destacar las fortalezas. Sin abordar puntualmente las inquie- tudes preparadas para la entrevista, alrededor de la computación en la nube y en la niebla, sus respuestas muestran el estado del país, más allá de estos nuevos desarrollos tecnológicos.

Revista Sistemas: ¿Cómo define usted la tecnología de la computación en el borde y en la niebla en Colombia?

Diego Molano Vega: Es un paso más en los avances tecnológicos y suministra nuevas oportunidades a un país como Colombia y facilita el acceso a los últimos desarrollos, más rápido y en forma más modular.

RS: ¿Cuál es el estado de Colombia en términos de infraestructura tecnológica? 
DMV: La mejor analogía para responder a su pregunta es la bicicleta, quien no pedalea, se cae. En otras palabras, es necesaria una permanente inversión para renovarla y esa debería ser la tendencia, tal y como sucede con los teléfonos celulares; por lo general, las personas cambian de equipo cada dos años, así que las empresas y el Gobierno no pueden dejar de invertir recursos y esfuerzos en ese cambio.

Por fortuna, las tecnologías disponibles en la nube permiten el acceso de los usuarios a lo más reciente, de acuerdo con las necesidades individuales.

\section{RS: Es inevitable no referirse a la} pandemia y la situación del país, ¿Colombia está en condiciones de hacer las inversiones necesarias en aras de fortalecer la infraestructura, para como dice usted pedaleary no caernos?

DMV: Hay que ver para qué y en dónde. Considerando las brechas que tiene Colombia, éstas son de muchos tipos. La primera relacionada con la infraestructura, medida a través de un índice del Foro Económico Mundial y del Portulans Institute -soy miembro de la Junta Directiva-, que ubican a nuestro país en el puesto 67 en términos de infraestructura tecnológica, por encima del promedio en América Latina; y establece en $70 \%$ los colombianos conectados a Internet. Con base en tales cifras y en lenguaje coloquial, el vaso se puede ver me- dio vacío o medio lleno. Yo lo veía medio lleno en mi gestión ministerial, lo que no quiere decir que se frene la inversión en fibra óptica para los hogares y, sobre todo, para los de estratos bajos.

RS: Ese 30\% que no está conectado a Internet pertenece a los estratos más bajos del país, grupo de población desprotegida y con grandes carencias, particularmente en esta pandemia. ¿De acuerdo?

DMV: Exactamente. Colombia está muy bien conectada internacionalmente, tiene 14 o 15 cables submarinos en el Pacífico y el Caribe y muy bien interconectada dentro del país. Nosotros pusimos fibra óptica y llegamos al $96 \%$ de los municipios, también trabajamos en redes de alta velocidad para llegar al resto de cabeceras municipales. En las autopistas que unen las ciudades estamos muy bien, nos falta mejorar en las callecitas de tales autopistas de la información, con el propósito de llegar a cada negocio, cada escuela y cada hospital. En otras palabras, trabajar sobre lo que llamamos el último kilómetro, en el que se requiere una fuerte inversión, para el despliegue de las redes $5 \mathrm{G}$.

RS: Y ¿la pandemia ha frenado ese recorrido? ¿Nos quedamos estáticos?

DMV: Las inversiones han continuado, a pesar de que hace un par de años tuvieron una frenada muy dura, ocasionada básicamente por el pago impuesto a las firmas Claro 
y Telefónica, por un Tribunal de Arbitramento. Si estas compañías tienen que pagar casi cinco billones de pesos, dinero que no se fue para el sector, sino para tapar el hueco fiscal, recursos que se dejaron de invertir en infraestructura para el sector de las telecomunicaciones, lo que generó una sensación de debilidad en el marco regulatorio. Han pasado varios años y se ha empezado a recuperar credibilidad, después de un buen tiempo en que no había confianza para invertir en el país. La acción en contra de esas dos compañías, sumada a la carga tributaria del sector, tan excesivamente alta -la telefonía celular paga cerca del $23 \%$ de IVA-, son medidas absurdas. Además, con una reforma tributaria anual, con unas reglas de juego sujetas a cambio con tanta frecuencia es difícil atraer inversión.

\section{RS: En ese contexto que usted describe isignifica que vamos pe- daleando, pero en reversa?}

DMV: Seguimos pedaleando, pero muchos países van más adelante que nosotros. Por ejemplo, México, Costa Rica y Uruguay que van avanzando rápido. México ahora tiene una muy buena oportunidad por la entrada en vigor del nuevo tratado de libre comercio con Estados Unidos y Canadá, país que tiene novedades en materia de Comercio Digital. Por otro lado, hay que considerar que en términos de infraestructura, no se contempla solamente lo que está en las calles, sino la que está dentro de las casas de los consumidores. La pandemia ha puesto al descubierto que en los hogares colombianos faltan buenas redes en el interior, además de una buena conexión wifi; y, sobre todo, computadores. ¿Cuántos hogares están habilitados para asumir la educación de los hijos en esas condiciones, particularmente cuando tienen tres estudiantes y un único equipo? Una casa típica colombiana está conformada por papá, mamá y dos o tres hijos. Las estadísticas muestran que todos están conectados a Internet, pero con un solo computador y eso no es suficiente.

\section{RS: Y ¿qué solución ve usted en el} corto plazo? ¿Alguna propuesta?

DMV: Hay que realizar acciones para reducir los impuestos en todo tipo de terminales de computación, tanto aranceles como IVA; bajar los precios de todos los equipos, como lo hicimos en su momento en la administración Santos, pero que al final de la misma se fue perdiendo por las reformas tributarias que iban eliminando esas ventajas. Así mismo, eliminar el IVA para todos los servicios de telecomunicaciones, esta es una forma de multiplicar la economía; cuando todos estamos en el mundo digital y el sector tiene ese tipo de cargas, la situación es muy difícil. Una pequeña ventaja hoy es que los servicios en la nube están exentos de IVA, pero las conversaciones apuntan a que en la próxima reforma tributaria se volverá a aplicar, hecho que sería gravísimo. 
RS: ¿Retomamos la descripción de las brechas?

DMV: La segunda brecha tiene que ver con que la gente está conectada, pero no, en la manera como usa la tecnología. Colombia comparada con países como Corea o algunos del norte de Europa, refleja un atraso en aumento. Aunque en la pandemia la situación ha mejorado, en Bogotá pasamos del 4\% de los teletrabajadores, al 25\%. De acuerdo con estudios del Centro Nacional de Consultoría, antes de la pandemia el $85 \%$ de los colombianos utilizaba Internet solo como una herramienta de comunicación básica o de entretenimiento, ahora lo hace en una forma más productiva.

La siguiente brecha tiene que ver con la forma en que el Gobierno usa la tecnología y ahí sí que el retraso es considerable. De liderar en 2014 los rankings de América Latina y de estar inclusive entre los países con mayor participación de la ciudadanía en el uso de servicios por medios electrónicos, el panorama hoy es muy distinto, vamos en descenso; de ocupar el puesto número 50 hoy estamos en la posición 67.

\section{RS: ¿Aqué se debe?}

DMV: A que el pedaleo al que me he referido debe ser permanente y en una forma innovadora, no siempre hay que hacer lo mismo. En el mundo digital vemos una tendencia muy válida en el ambiente de Startups y encontramos muchas firmas colombianas; los emprendedores prestan servicios a las entidades del Estado y es necesario contemplar la forma de ayudarlas para dar paso al ofrecimiento de varios servicios como sistemas de data analytics para el Estado, seguridad y ciberseguridad entre otros. En esa dirección no vamos al mismo ritmo del mundo, no significa que estemos detenidos, pero no vamos tan rápido como deberíamos.

\section{RS: En su opinión ¿cómo está la empresa privada?}

DMV: Las empresas privadas no se están transformando digitalmente a la velocidad que deberían hacerlo, como lo están haciendo, por ejemplo, Asia y el norte de Europa. Hay algunos sectores en que vamos bien, como el financiero.

\section{RS: Supongo que se refiere a las pequeñas y medianas empresas, ¿verdad?}

DMV: Sí y considerando que en ese sector está la gran mayoría de empleos, la situación es preocupante. Si no hay transformación digital desaparecerán con rapidez y serán reemplazados por modelos de negocios extranjeros. En el mundo digital no existen fronteras y ante esa carencia, los nuevos empleos serán cubiertos desde afuera del país. Hecho directamente relacionado con la brecha más grande que tenemos alrededor de las necesidades de talento digital, por encima de la infraestructura, del uso del Gobierno de los medios digitales para ofrecer servicios o si las 
empresas se están o no transformando. Específicamente, la necesidad apunta a cuatro tipos de talento. El primero compuesto por programadores y está representada en más de 100.000 en el corto plazo.

\section{RS: Pero ¿cómo, si los estudiantes de ingeniería de sistemas ya no quieren ser programadores?}

DMV: Esa situación es muy preocupante. El segundo talento se basa en la necesidad de que todos los profesionales tengan un poquito de ingenieros de sistemas. A los comunicadores, por ejemplo, se les pregunta ¿cómo funciona el algoritmo de la publicidad de Google?, y no tienen ni idea. En otras palabras, estamos formando profesionales para el siglo pasado. Hay que formar ingenieros de sistemas, pero insisto en las habilidades que exige este nuevo mundo. En la universidad Área Andina, de la cual soy presidente, lo denominamos 'humanismo digital'. El tercer talento está representado en las mujeres -a propósito, me alegra muchísimo que la Junta Directiva de Acis esté conformada por mujeres-. Es inminente que masivamente ellas salgan a trabajar; no se trata de un asunto de igualdad de género, es que necesitamos una economía más productiva y las mujeres cuentan con mayores habilidades para esa transformación, que los hombres.

\section{RS: ¿Porqué?}

DMV: Tienen más habilidades blandas que los hombres; es decir, de empatía, de comunicación, que en nuestra cultura los hombres no las hemos desarrollado.

Y el cuarto talento se basa en la formación de líderes para esta revolución. Y no los tenemos. Busquemos cinco candidatos para ser ministros en la transformación digital desde sus respectivos sectores y no se encuentran.

\section{RS: ¿Y cuál es la solución? Pare-} ciera que estamos en un círculo vicioso y sin salida.

DMV: La solución está en que cada uno de nosotros nos apersonemos del asunto; no se trata de que 'papá' Gobierno venga a hacerlo. Las oportunidades son visibles para programadores y líderes, están a la mano. Y quien adquiera esas habilidades encontrará sin dificultad oportunidades laborales y sus negocios serán más exitosos.

\section{RS: Hablemos ahora sobre el mar-} co regulatorio que soporta la tecnología en Colombia. ¿Qué opina al respecto?

DMV: Antes era un marco regulatorio de telecomunicaciones muy específico, hoy en día es transversal y contempla diferentes sectores como el financiero, el e-commerce, la salud para regular la historia clínica electrónica, la justicia para promover el expediente judicial electrónico, otro laboral que promueve el teletrabajo. Ahora el de las telecomunicaciones es el menos relevante y se trata de una la- 
bor conjunta, no de un único ministerio.

RS: Poco hablamos de los asuntos inherentes al tema central de la revista: la computación en el borde y en la niebla, pero teniendo en cuenta su condición de exministro de las TIC, son importantes otros temas. Por ejemplo, la seguridad y la ciberseguridad. En su opinión ¿cómo está el país al respecto?

DMV: Ahí también carecemos de talento. Necesitamos más profesionales que manejen estos asuntos, pero también que los ciudadanos seamos conscientes de los riesgos relacionados con la ciberseguridad. $Y$ hay que avanzar en dos frentes alrededor de la capacitación. Por un lado, disponer de profesionales que ayuden al Gobierno y al sector privado para evitar eventos y proteger los sistemas de información. Pero, también informar a los ciudadanos y capacitarlos sobre las buenas prácticas en búsqueda de la seguridad.

RS: Ahora sí sobre el tema central de esta edición. ¿Colombia puede aprovechar el ambiente de la computación en el borde y en la niebla?. ¿es posible habilitar esa promesa de valor de la cuarta revolución industrial?

DMV: Claro que sí, pero con gente capacitada.

Sara Gallardo M. Periodista comunicadora, universidad Jorge Tadeo Lozano. Ha sido directora de las revistas Uno y Cero, Gestión empresarial y Acuc Noticias. Editora de Aló Computadores del diario El Tiempo. Redactora en las revistas Cambio 16, Cambio y Clase Empresarial. Coautora del libro "Lo que cuesta el abuso del poder". Ha sido corresponsal de la revista Infochannel de México; de los diarios La Prensa de Panamá y La Prensa Gráfica de El Salvador y corresponsal de la revista IN de Lanchile e investigadora en publicaciones culturales. Se ha desempeñado también como gerente de Comunicaciones y Servicio al Comensal en Inmaculada Guadalupe y amigos en Cía. S.A. (Andrés Carne de Res) y editora de Alfaomega Colombiana S.A.; es editora de esta revista. 